\title{
General Reflection Coefficients for Acoustic Multipole Sources on guided waves in Isotropic Tubular Structure Liquid Metal Sodium Filled
}

\author{
Li ZHANG \\ School of Physics, Tonghua Normal University, China
}

KEYWORD: Guided waves, Isotropic tubular structure, General reflection coefficients

\begin{abstract}
A new and effective analytical method is presented for the acoustic multipole sources in isotropic tubular structure filling liquid metal sodium whose symmetric principal axis is parallel to the tube axis the exact solutions could be found. In this paper, the new method is adopted to simulate the general reflection coefficients in isotropic tubular structure filling liquid metal sodium for the first time. By selecting a group of displacement potentials and a cylindrical coordinate system oriented along the tube axis, the solutions of the multipole acoustic field are derived for isotropic tubular structure filling liquid metal sodium. The acoustic fields inside and outside the tube excited by a multipole source are investigated. The general reflection coefficients in the tube are numerically simulated. It is found that the general reflection coefficients in isotropic tubular structure filling liquid metal sodium whose symmetric principal axis is parallel to the tube axis inside the tube excited by monopole, dipole sources and quadrupole source are change as the change of thickness of tube and temperature.
\end{abstract}

\section{INTRODUCTION}

It has been found that wave propagation in an anisotropic medium has become a more active research topic. The more interesting of the anisotropic formation study is on the transversely isotropic medium (TIM). This is mainly due to PTL (periodic thin-layer) and EDA (extensivedilatancy anisotropy) models, which are important to the reservoir rocks and are all equivalent to the transversely isotropic formation in the hypothesis of the long wavelength. Along with the development of well sources technology to study acoustic wave propagation in anisotropic medium become more important for acoustics logging. If the medium outside the borehole is TIM, the wave field solution can be analytically solved only in the condition of the symmetric principal axis of TIM is parallel to the borehole axis. To study the wave-field characteristics in an important case when the symmetric principal axis of TIM is not parallel to the borehole axis, some approximation methods has been presented. Ellefsen, Sinha and Norris presented a perturbation method and investigated the influence of weak elastic anisotropic on the tube wave speed [1-5]. However, their perturbation methods cannot be used to investigate the characteristic of the full-wave as well as $\mathrm{P}$ and $\mathrm{S}$ waves. Zhang et al. [6-9] presented another perturbation method that can treat the full-wave acoustic field for TIM with the principal axis perpendicular to the borehole axis. They obtained zero- and first- order approximation solutions for the acoustic fields inside and outside the borehole. This method can be possibly used to analytically solve the shear wave splitting in the borehole. Zhang et al. [10] investigated frequency-wave number domain of this perturbation method for acoustical multipole sources in transversely isotropic medium.

In this Paper, we will give an effective analytical method is presented for the acoustic multipole sources in isotropic tubular structure filling liquid metal sodium whose symmetric principal axis is parallel to the tube axis although the exact solutions. The characteristic of each order of the perturbation solutions outside the tube are discussed when the radial variable $r$ is changed. The acoustic fields inside and outside the borehole excited by a multipole source are investigated. The dispersion characteristics excited by monopole, dipole sources and quadrupole source are change as the change of thickness of tube and temperature.

\section{FORMULATION}

Consider a fluid-filled tube surrounded by tubular structure whose symmetric principal axis is parallel to the borehole axis. The density and velocity of the fluid inside the borehole are $\rho \mathrm{f}$ and Vf, respectively, and $\mathrm{R}$ is the radius of the tube. We adopt a cylindrical coordinate system $(\mathrm{r}, \theta, \mathrm{z})$ 
centered at the center of multipole source and oriented along the tube axis, and define a Cartesian reference frame $(\mathrm{x}, \mathrm{y}, \mathrm{z})$ whose $\mathrm{z}$ axis is parallel to the tube axis.

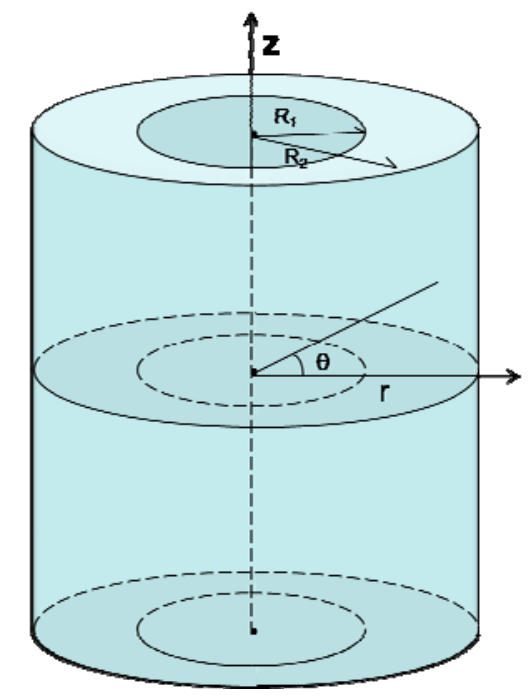

Fig. 1 The framework model

In the Cartesian coordinate system (x,y, z), the displacement $\vec{U}$ in the isotropic tubular structure outside the tube satiş,ies

$$
U=\nabla \phi+\nabla \times\left(\chi e_{z}\right)+\nabla \times \nabla \times\left(\psi \overrightarrow{e_{z}}\right)
$$

The equation in Eq. (1) is the field equation in isotropic medium. If the acoustic field is excited by an nth-order multipole source at $\mathrm{r}=0$, the zero-order displacement potentials in the frequency wavenumber $(\omega, \mathrm{kz})$ domain satisfying the radiation

(2)

$$
\begin{aligned}
& \phi_{n}\left(r, \theta, k_{z}, \omega\right)=-\frac{V_{0}(\omega)}{2 \pi} \frac{1}{n !}\left(\frac{v r_{0}}{2}\right)^{n}\left[B_{n} K_{n}\left(v_{p} r\right)+C_{n} I_{n}\left(v_{p} r\right)\right] \cos n\left(\theta-\theta_{0}\right), \\
& \chi_{n}\left(r, \theta, k_{z}, \omega\right)=-\frac{V_{0}(\omega)}{2 \pi} \frac{1}{n !}\left(\frac{v r_{0}}{2}\right)^{n}\left[D_{n} K_{n}\left(v_{s} r\right)+E_{n} I_{n}\left(v_{s} r\right)\right] \sin n\left(\theta-\theta_{0}\right), \\
& \psi_{n}\left(r, \theta, k_{z}, \omega\right)=-\frac{V_{0}(\omega)}{2 \pi} \frac{1}{n !}\left(\frac{v r_{0}}{2}\right)^{n}\left[F_{n} K_{n}\left(v_{s} r\right)+G_{n} I_{n}\left(v_{s} r\right)\right] \cos n\left(\theta-\theta_{0}\right) .
\end{aligned}
$$

condition at infinity can be written as[10-14]

Where $\mathrm{Kn}$ is the second kind of nth-order modified

$$
v_{s}=\sqrt{k_{z}^{2}-k_{s}^{2}} \quad k_{p}^{2}=\rho \omega^{2} /(\lambda+2 \mu)
$$

Bessel function, r0 is the multipole source separation and are the weighting coefficients.The $B_{n}, C_{n}, D_{n}, E_{n}, F_{n}, G_{n} \quad k_{s}^{2}=\rho \omega^{2} / \mu$

acoustical potential in a tube for an nth multipole source can be written the same form as that in the isotropic medium [7-14]

$\varphi_{n}^{f}\left(\omega, \mathrm{k}_{\mathrm{z}}\right)=-\frac{1}{4 \pi^{2} n !}\left(\frac{v r_{0}}{2}\right)^{n}\left[\varepsilon_{n} K_{n}(v r) \cos n\left(\theta-\theta_{0}\right)+A_{n} I_{n}(v r) \cos n\left(\theta-\theta_{0}\right)\right]$

$v=\sqrt{k_{z}^{2}-\omega^{2} / V_{f}^{2}}$ 
Where, $V_{f}$ is the acoustic velocity of the fluid inside the tube, $\varepsilon \mathrm{n}=2-\delta \mathrm{n} 0$ is Neumann' $\mathrm{s}$ factor, r0 is the multipole source separation, In is the first kind of the nth-order modified Bessel function, and An is the reflection coefficient which can be determined by the boundary conditions at tube wall.

$U_{r}^{I}=U_{r}^{I I}$,

$-P_{f}^{I}=\tau_{r r}^{I I}$,

$0=\tau_{r z}^{I I}$,

$0=\tau_{r \theta}^{I I}$,

The boundary conditions at $r=R_{1}$ are [6-8, 10-12]

$U_{r}^{I}=U_{r}^{I I}$,

$-P_{f}^{I}=\tau_{r r}^{I I}$,

$0=\tau_{r z}^{I I}$,

$0=\tau_{r \theta}^{I I}$,

The boundary conditions at $r=R_{2}$ are [6-8, 10-12]

$\tau_{r r}^{I I}=0$,

(5)

$\tau_{r z}^{I I}=0$,

$\tau_{r \theta}^{I I}=0$,

Where the superscripts I and II represent the media inside and outside the tube. Substituting the fields inside and outside the tube into Eqs. (4) and

, yields a linear equati ${ }^{G_{n}}$ on group about and. By this linear equation, we can calculate all unknown coefficients inside and outside the tube. Then, it is easy to analyze the acoustic field characteristics by the numerical simulation.

$A_{n}, B_{n}, C_{n}, D_{n}, E_{n}, F_{n}$

\section{NUMERICAL SIMULATION}

In numerical simulation, the multipole source separation radius is taken to be $0.01 \mathrm{~m}$, inside of the tube radius $R_{1}$ is taken to be $4 \mathrm{~m}$, outside of the tube radius $R_{2}$ are taken to be $4.01 \mathrm{~m}, 4.02 \mathrm{~m}$, $4.03 \mathrm{~m}, 4.04 \mathrm{~m}$ and $4.05 \mathrm{~m}$, respectively. Three groups of the parameters are given in Table 1 .

Table1 Three groups of the parameters

\begin{tabular}{|c|c|c|}
\hline Note & densities of the fluid sodium & velocity of the fluid sodium \\
\hline 1 (Temperature=900K) & $750 \mathrm{~kg} / \mathrm{m}^{3}$ & $2097 \mathrm{~m} / \mathrm{s}$ \\
\hline densities of the isotropic tubular structure & the shear wave velocity & P-wave velocity \\
\hline $7930 \mathrm{~kg} / \mathrm{m}^{3}$ & $4000 \mathrm{~m} / \mathrm{s}$ & $5758 \mathrm{~m} / \mathrm{s}$ \\
\hline
\end{tabular}

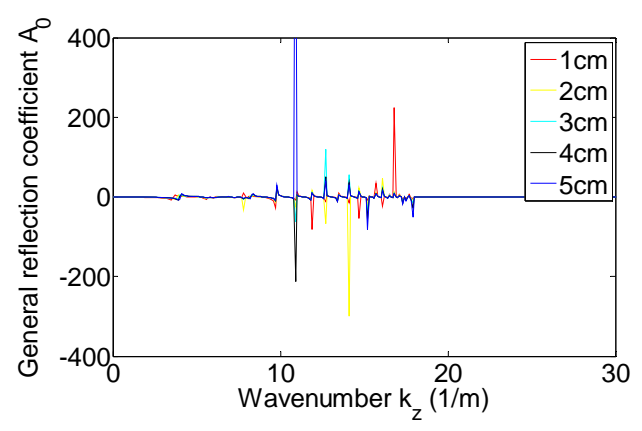

(a) 


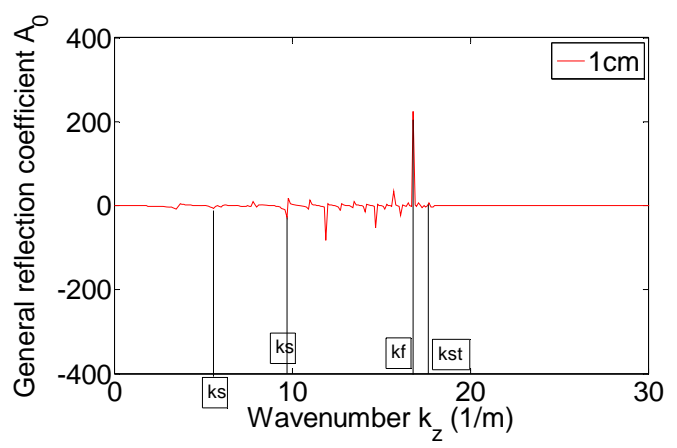

(b)

Fig.2 General reflection coefficients $\mathrm{A} 0$ for $\mathrm{f}=6000 \mathrm{~Hz}$ as a function of the wavenumber $k_{\mathrm{z}}$

(Thickness of tube are $1 \mathrm{~cm}, 2 \mathrm{~cm}, 3 \mathrm{~cm}, 4 \mathrm{~cm}$ and $5 \mathrm{~cm}$, Temperature $=900 \mathrm{~K}$ )

In the numerical simulations, the displacement potentials $(\phi, \psi, \chi)$ inside the borehole and the reflection coefficients $\left(A_{n}\right)$ outside borehole were studied, Figure 2 displays the relationship between the general reflection coefficient (An) and the wavenumbers $\left(k_{z}\right)$ for a monopole source (n $=0)$ at a frequency of $6000 \mathrm{~Hz}$. For the isotropic medium, the wavenumbers of the press wave, shear wave, fluid wave and guided wave are 6.54, 9.42, 17.96 and 18.06, respectively. We labeled these values on the wavenumber axis in Fig. 2 (b), as kp, ks, kf and kst, respectively.

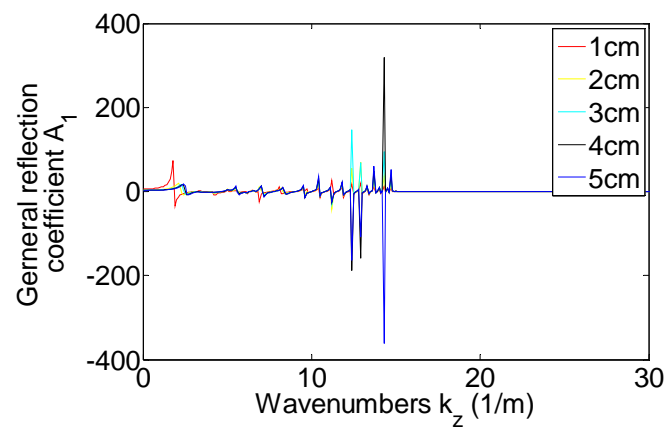

(a)

Figure 3 presents the simulated results for a dipole source $(n=1)$. The frequencies for the numerical investigations of the dipole sources were set to $5000 \mathrm{~Hz}$. For the isotropic medium, the wavenumbers of the press wave, shear wave, fluid wave and guided wave are 5.45, 7.85, 14.97 and

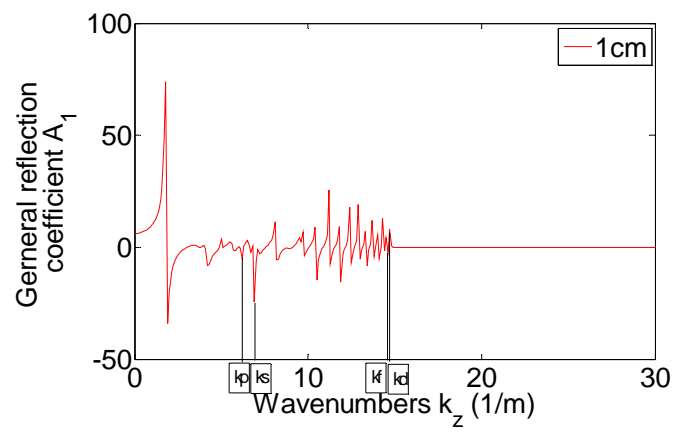

(b)

Fig.3 General reflection coefficients A1 for $\mathrm{f}=5000 \mathrm{~Hz}$ as a function of the wavenumber $k_{\mathrm{z}}$ (Thickness of tube are $1 \mathrm{~cm}, 2 \mathrm{~cm}, 3 \mathrm{~cm}, 4 \mathrm{~cm}$ and $5 \mathrm{~cm}$, Temperature=900K) 
14.99, respectively. We labeled these values on the wavenumber axis in Fig. 3 (b), as $k_{\mathrm{p}}, k_{\mathrm{s}}, k_{\mathrm{f}}$ and $k_{\mathrm{d}}$, respectively.

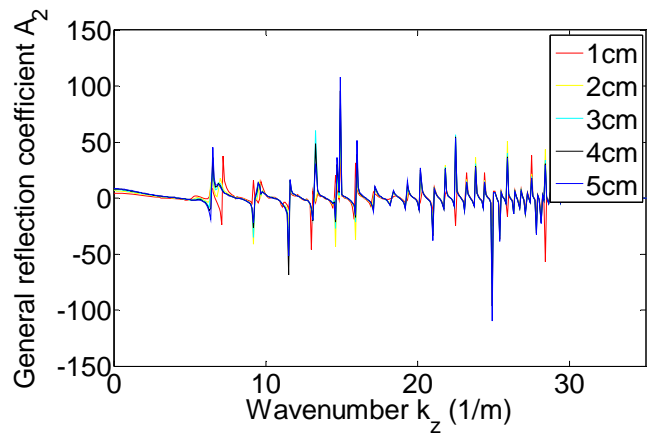

(a)

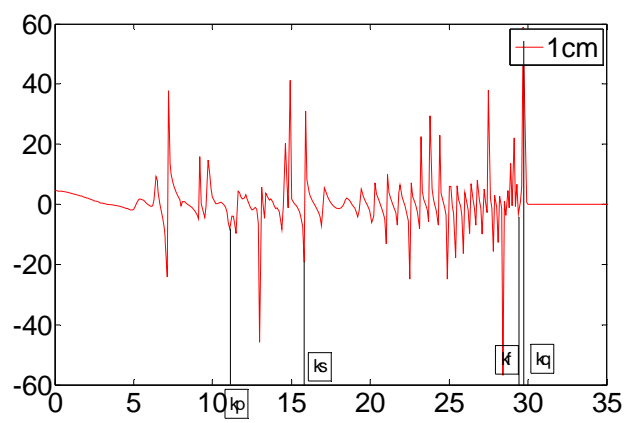

(b)

Fig.4 General reflection coefficients A2 for $\mathrm{f}=10000 \mathrm{~Hz}$ as a function of the wavenumber $k_{\mathrm{z}}$

(Thickness of tube are $1 \mathrm{~cm}, 2 \mathrm{~cm}, 3 \mathrm{~cm}, 4 \mathrm{~cm}$ and $5 \mathrm{~cm}$, Temperature $=900 \mathrm{~K}$ )

Figure 4 presents the simulated results for a quadrupole source $(n=2)$. The frequencies for the numerical investigations of the quasi-pole sources were set to $10000 \mathrm{~Hz}$. For the isotropic medium, the wavenumbers of the press wave, shear wave, fluid wave and guided wave are 10.906, 15.7, 29.94 and 29.99, respectively. We labeled these values on the wavenumber axis in Fig. 4 (b), as kp, $\mathrm{ks}, \mathrm{kf}$ and kq, respectively.

The five solid lines in Figure 2 represent for the result of exact solution for the isotropic tubular structure in parameter group 1, outside of the tube radius $R_{2}$ are taken to be $4.01 \mathrm{~m}, 4.02 \mathrm{~m}, 4.03 \mathrm{~m}$, $4.04 \mathrm{~m}$ and $4.05 \mathrm{~m}$, that is thickness of tube are $1 \mathrm{~cm}, 2 \mathrm{~cm}, 3 \mathrm{~cm}, 4 \mathrm{~cm}$ and $5 \mathrm{~cm}$. It is found that the general reflection coefficients in isotropic tubular structure filling liquid metal sodium whose symmetric principal axis is parallel to the tube axis inside the tube excited by monopole source are change as the change of thickness of tube.

\section{SUMMARY}

In summary, the guide waves excited by a monopole, dipole, and quadrupole sources in a fluidfilled sodium tube surrounded by a isotropic tubular structure with principal axis parallel to the tube axis are studied by the method. It is found that the general reflection coefficients in isotropic tubular structure filling liquid metal sodium whose symmetric principal axis is parallel to the tube axis inside the tube excited by multipole source are change as the change of thickness of tube and temperature. It shows that the method is a feasible method and can be applied to the complicated anisotropic tubular structure in acoustic multipole sources.

\section{ACKNOWLEDGEMENT}

This research was financially supported by the Project of Education Department of Jilin Province (Grant No.2014388) 


\section{REFERENCES}

[1] Ellefsen K J, Cheng C H and Tubman K M, Estimating phase velocity and attenuation of guided waves in acoustic logging data, Geophysics.54 (1989) 1054-1059.

[2] Sinha B K, Elastic waves in crystals under a bias, Ferroelectrics. 41 (1982) 61-73.

[3] Norris A N, Sinha B K and Kostek S, Acoustoelasticity of solid/ fluid composite systems, Geophys. J. Int.118 (1994) 439-446.

[4] Sinha B K, Norris A N and Chang S K, Borehole flexural modes in anisotropic formations, Geophysics. 59(1994) 1037-1052.

[5] Norris A N, Sinha B K, The speed of a wave along a fluid-solid interface in the presence of anisotropy and prestress, J.Acoust.Soc.Am.98 (1995) 1147-1154.

[6] Zhang Li, Zhang Bixing and Wang Kexie, Perturbation analysis on guided waves in a fluidfilled borehole surrounded by a cubic crystal anisotropic medium, CHIN. PHYS.LETT. 24 (2007) 3179-3183

[7] Zhang B X and Wang K X, Theoretical study of perturbation method for acoustic multipole logging in anisotropic formation, J. Acoust. Soc. Am.99 (1996) 2674-2685.

[8] Zhang B X and Wang K X, Theoretical study of multipole acoustic logging in anisotropic twophase medium formation, Chinese Journal of Geophysics. 43 (2000) 707-718.

[9] Zhang B X, Wang K X, Multipole sources in a fluid - filled borehole surrounded by a transversely isotropic elastic solid. 43 (2000) 707-718.

[10] L. Zhang, X. F. Liu, W. Yuan and X. T. Wang, Analytical Perturbation Methods for Studying a Transversely Isotropic Medium in Multipole Acoustic Logging, Journal of the Korean Physical Society. 64 (2014) 1808-1813.

[11] Zhang B X, Dong H F and Wang K X, Multipole sources in a fluid - filled borehole surrounded by a transversely isotropic elastic solid, J. Acoust. Soc. Am.96 (1994) 2546-2555.

[12] Kurkjian A L and Chang S K, Acoustic multipole sources in fluid - filled borehole, Geophysics. 51 (1986) 148-163.

[13] Schmitt D P, Cheng C H and Toksoz M N, Shear wave logging in saturated porous formations, SPWLA, 28th Annual Logging Symposium, 1987

[14] Schmitt D P, Acoustic multipole logging in transversely isotropic poroelastic formations, J. Acoust. Soc. Am.86 (1989) 2397-2421. 